\title{
LncRNA SNHG16 contributes to osteosarcoma progression by acting as a ceRNA of miR-1285-3p
}

\author{
Xiao Xiao ${ }^{1,2+}{ }^{+}$Ge Jiang ${ }^{3 \dagger}$, Shengtao Zhang ${ }^{4}$, Shuo Hu ${ }^{1,2}$, Yunshan Fan ${ }^{1,2}$, Gang Li ${ }^{1,2}$, Haiyang Yu ${ }^{1,2}$ and \\ Shisheng $\mathrm{He}^{1,2^{*}}$
}

\begin{abstract}
Background: The long non-coding (Inc) RNA activated by small nucleolar RNA host gene 16 (SNHG16), which has been reported to play a vital role in a number of different types of cancer, is a novel IncRNA. However, following an osteosarcoma (OS) study, the expression pattern, biological roles, clinical values and potential molecular mechanism of SNHG16 remain unclear. In the current study, we aimed to examine its expression and possible function in osteosarcoma (OS).

Method: Cell proliferation was measured by colony formation assay and Cell Counting Kit-8 (CCK-8) in vitro, and xenograft transplantation assay in vivo. Meanwhile, we used transwell chambers to test cell migration and invasion was evaluated. Cell cycle and apoptosis was evaluated by flow cytometry assay. Immunoblotting and qPCR analysis was carried out to detect protein and gene expression, respectively. Luciferase reporter assay was used to predict the potential downstream genes.

Results: The present study demonstrated that SNHG16 is highly expressed in both the tissues of patients with OS, as well as OS cell lines, and its expression level was positively correlated with clinical stage and poor overall survival. Functional assays revealed that the depletion of SNHG16 inhibits OS growth, OS cell progression and promotes apoptosis both in vivo and in vitro. In addition, the present study revealed that microRNA-1285-3p expression levels can be decreased by SNHG16 acting as a 'sponge', and that this pathway takes part in OS tumor growth in vivo, and OS cell proliferation, invasion, migration and apoptosis in vitro.

Conclusions: The results from the present study demonstrate the role of IncRNA SNHG16 in OS progression, which is SNHG16 might exert oncogenic role in osteosarcoma (OS) by acting as a ceRNA of miR-1285-3p, and it may become a novel target in OS therapy.
\end{abstract}

Keywords: Long non-coding RNA, Small nucleolar RNA host gene 16, Osteosarcoma, Progression, Competing endogenous RNA, microRNA-1285-3p

\footnotetext{
* Correspondence: shsyhss@163.com

'Xiao Xiao and Ge Jiang contributed equally to this work.

'Department of Orthopedic, Shanghai Tenth People's Hospital, Tongji

University School of Medicine, Shanghai 200072, China

${ }^{2}$ Spinal Pain Research Institute, Tongji University School of Medicine,

Shanghai 200072, China

Full list of author information is available at the end of the article
}

(C) The Author(s). 2021 Open Access This article is licensed under a Creative Commons Attribution 4.0 International License, which permits use, sharing, adaptation, distribution and reproduction in any medium or format, as long as you give appropriate credit to the original author(s) and the source, provide a link to the Creative Commons licence, and indicate if changes were made. The images or other third party material in this article are included in the article's Creative Commons. licence, unless indicated otherwise in a credit line to the material. If material is not included in the article's Creative Commons licence and your intended use is not permitted by statutory regulation or exceeds the permitted use, you will need to obtain permission directly from the copyright holder. To view a copy of this licence, visit http://creativecommons.org/licenses/by/4.0/. The Creative Commons Public Domain Dedication waiver (http://creativecommons.org/publicdomain/zero/1.0/) applies to the data made available in this article, unless otherwise stated in a credit line to the data. 


\section{Background}

Osteosarcoma (OS) is a type of bone malignancy that most frequently occurs in children and young adults, and is associated with highly aggressive and unfavorable prognoses [1]. Previous studies have indicated that risk factors such as genetic mutations and transcriptional regulatory disorders are key to the development of OS [2]. Treatment including surgical excision, radiotherapy and neoadjuvant chemotherapy has been achieved, but the prognosis of patients with advanced clinical stages at diagnosis remains unfavorable $[3,4]$. Therefore, the molecular mechanism underlying OS progression and new molecular biomarkers for early diagnosis require further clarification in order to improve the diagnosis and effective therapy at the onset of the disease.

Long non-coding RNAs (lncRNAs) are a type of RNA of $>200$ nucleotides in length, are not protein coding, and play critical roles in the regulation of cell proliferation, apoptosis, migration, invasion, the cell cycle, drug resistance and chromatin remodeling [3-6]. Over the last few years, lncRNAs have gradually been recognized as biological factors in OS. For example, the growth and metastasis of osteosarcoma are promoted by long-noncoding RNA SNHG5 via sponging the miR-212-3p/ SGK3 axis [7]. Silencing of lncRNA ANCR suppresses the migration and invasion of osteosarcoma cells by activating the p38MAPK signalling pathway [8]. Furthermore, the resistance of doxorubicin in OS was enhanced by IncRNA FOXC2-AS1 [3]. However, the role of lncRNA in OS pathogenesis has not yet been clearly defined.

Small nucleolar RNA host gene 16 (SNHG16) was originally identified as an oncogene in neuroblastoma, with a poor patient outcome when increased levels of SNHG16 were expressed [9]. Meanwhile, high expression levels of SNHG16 were negatively associated with overall survival time in laryngeal squamous cell carcinoma [10], bladder cancer [11], esophageal squamous cell carcinoma [12] and endometrial carcinoma [13]. Consistent with the outcomes of clinical samples, SNHG16 exhibits a tumorigenic phenotype in cervical [14] and colorectal cancer cell lines in vitro [15]. Finally, knockdown of SNHG16 improved chemosensitivity in bladder cell lines, suggesting that interfering SNHG16 may decrease chemoresistance in patients with bladder cancer [16]. Nevertheless, the potential molecular mechanism and functional role of SNHG16 in OS remains unknown.

miRNAs are endogenous small noncoding RNAs (1722 nucleotides in length), which take part in tumorigenesis via combination with the 3 ' untranslated region (3'UTR) of target genes to interfere with transcription or inhibit translation [17]. Upregulation of miR-1285 could suppress renal carcinoma invasion and migration via regulating TGM2 expression [18], and it can also suppress breast cancer proliferation by regulating TMEM194A expression [19]. The present study confirmed that miR-1285-3p worked as the suppressor gene in OS cell lines and tissues; in constrast, SNHG16 was highly expressed in OS and promoted its progression. Since the theory about competing endogenous RNA (ceRNA) between IncRNA and miRNA is widely accepted [20], the present study demonstrated that SNHG16 contributes to OS progression via acting as the ceRNA of miR-1285-3p.

\section{Methods}

\section{Clinical samples}

A total of 50 patients with OS were recruited into the present study. OS tissues and paired adjacent normal tissues were obtained from the First Affiliated Hospital of Nanchang University (Nanchang, China). All resected specimens were stored at $-80^{\circ} \mathrm{C}$ prior to RNA extraction. The present study was reviewed and approved by the Ethics Committee of the First Affiliated Hospital of Nanchang University. All the informed consent was written by patients, and the parent/guardian of patients who were $<18$ years old.

\section{Cell culture}

Human OS cell lines (U2OS, MNNG/HOS, 143b, SJSA and MG63), 293 cell line and a normal osteoblast cell line (hFOB 1.19) were obtained from the Cell Bank of the Chinese Academy of Sciences. All cell types were cultured in DMEM (Hyclone; Thermo Fisher Scientific, Inc.) supplemented with $10 \%$ fetal bovine serum (FBS) (Gibco; Thermo Fisher Scientific, Inc.) at $37^{\circ} \mathrm{C}$ in $5 \%$ $\mathrm{CO}_{2}$ and $95 \%$ air.

\section{Plasmid construction}

The SNHG16 fragment containing the miR-1285-3p binding site was amplified and cloned into the Notl and XhoI restriction sites of the psiCHECK2 Vector (Promega Corp) in order to obtain the reporter vector psiCHECK2-SNHG16-wild-type (SNHG16-wt). Mutating the miR-1285-3p binding sites in SNHG16 resulted in the psiCHECK2-SNHG16-mutant-type vector (psiCHECK2-SNHG16-mut).

\section{Cell transfection}

Two different small interfering (si)RNAs against SNHG16 were designed and synthesized by GenePharma, and transfected into U2OS and MNNG/HOS cells using Lipofectamine ${ }^{\curvearrowleft} 3000$ (Invitrogen; Thermo Fisher Scientific, Inc.) according to the manufacturer's protocol. Cells were collected $48 \mathrm{~h}$ after transfection, and the knockout efficiency was detected via reverse transcription-quantitative PCR (RT-qPCR). The 
sequences of SNHG16 siRNA were as follows: SNHG16 siRNA, forward, 5' -CCUGGGUAUAAUCUCACAATT$3 '$, and reverse, 5'-UUGUGAGAUUAUACCCAGGTT3'; SNHG16 siRNA, forward, 5' -GGAACAUACUGCUA UCAUATT-3', and reverse, 5'-UAUGAUAGCAGUAU GUUCCTT-3'; miR-1285-3p mimic, forward, 5' UCUGGGCAACAAAGUGAGACCU-3', and reverse, 5'-GUCUCACUUUGUUGCCCAGAUU-3'; miR-1285$3 p$ inhibitor, forward, $5^{\prime}$-AGGUCUCACUUUGUUGCC CAGA-3'.

\section{RT-qPCR}

Total RNA was extracted from OS tissues of cells using TRIzol ${ }^{\circ}$ reagent. RT was performed using PrimeScript $^{\mathrm{mm}} \mathrm{RT}$ reagent kit (Takara Bio). The RT conditions were $15 \mathrm{~min}$ at $37{ }^{\circ} \mathrm{C}$ and $5 \mathrm{~s}$ at $85^{\circ} \mathrm{C}$, and the mixture was subsequently stored at $4{ }^{\circ} \mathrm{C}$. The qPCR primers used were as follows: U6 forward, $5^{\prime}$ - TTAC ATTGCTATCCACAGAACGG - 3', and reverse, 5'CTATGCTGCTGCTTTTTGCTC-3'; miR-1285-3p forward, 5'-GCGTCTGGGCAACAAAGTG-3', and reverse, 5'-AGTGCAGGGTCCGAGGTATT-3'; $18 \mathrm{~S}$ forward, 5'-GAAACGGCTACCACATCC - 3', and reverse, 5'-ACCTCCCGTTCAGACCA-3'; SNHG16 forward, 5'-TACTCTGTTGGAAGAGCCTAA-3', and reverse, 5'-GGGTGTTGGTAACGAAA $-3{ }^{\prime}$. By the way, the short length of miRNA is not suitable for the use of bidirectional primers, we use RT-primer to prolong their sequence and quantify them. The reverse transcription primer sequence is composed of a 5 '-end stem-loop structure and a 3 '-end specific sequence. The primer sequence is about 42-44 nucleotides; the 36 nucleotide sequence at its 5 'end is fixed as stem-loop, and the 6-8 nucleotide structure at its 3 ' end are complementary to microRNA. Firstly, we can get the mature sequence of microRNA through miRbase (http://www.mirbase.org). Then, we download the sequence and put them into the "miRNA RT primer", a primer design software, to get the reverse primer sequence. Finally, the reverse and the forward primer are producted by primer3plus, an online primer design website (http://www.primer3plus.com).

\section{Western blotting}

OS tissues and cells were harvested and then split using RIPA protein extraction reagent (Beyotime Institute of Biotechnology). Proteins were transferred onto PVDF membranes, and then incubated with the specific primary and secondary antibodies. Autoradiogram and chemiluminescence were used to quantify the densitometry, and GAPDH was used as the control. Antibodies GAPDH, pro-caspase3, cleaved-caspase3, bcl-2 and bax were purchased from Abcam.

\section{Cell proliferation assays}

The change in cell viability following transfection was assessed using a Cell Counting Kit-8 (CCK-8; Dojindo Molecular Technologies) assay. In brief, OS cell lines were equally seeded into 96-well plates following treatment with different types of siRNA, and the cells were cultured with CCK8 reagent for $2 \mathrm{~h}$. The optical density (OD) was measured every $24 \mathrm{~h}$ using at $450 \mathrm{~nm}$, according to the manufacturer's protocol.

\section{Cell migration and invasion assays}

In the cell migration and invasion assays, Transwell chambers were used (Costar; Corning, Inc.). For the migration assay, $5 \times 10^{4}$ cells were added to the upper chambers with $200 \mu \mathrm{l} \mathrm{FBS-free} \mathrm{medium,} \mathrm{while} 500 \mu \mathrm{l}$ medium supplemented with 10\% FBS was injected into the lower chambers. For the invasion assay, the upper chamber was pre-coated with Matrigel (BD Biosciences), and $1 \times 10^{5}$ cells were plated in the upper chamber cultivated in $200 \mu \mathrm{l} \mathrm{FBS-free} \mathrm{medium.} \mathrm{The} \mathrm{lower} \mathrm{chamber}$ was filled with complete medium. After $24 \mathrm{~h}$ incubation, the traversed cells were stained with crystal violet. All images are observed under the microscope (Leika) and taken by Leica LAS AF Lite software. The image resolution is at least 300ppi of each image.

\section{Colony formation assay}

Cells $\left(1 \times 10^{3} /\right.$ well $)$ were seeded in 12 -well plates and incubated in complete medium. The colonies were fixed with $10 \%$ formaldehyde for $10 \mathrm{~min}$ and stained with $1 \%$ crystal violet after 2 weeks. The colony number was counted manually.

\section{Cell cycle and apoptosis analysis}

In the cell cycle analysis, propidium iodide (PI) was used to stain OS cells, and a Cycle Reagent kit (BD Biosciences) was used for the analysis. In the apoptotic analysis, cells were stained with PI and Annexin V-FITC. Flow cytometric analysis was performed with a flow cytometer (BD Biosciences) $48 \mathrm{~h}$ after cell transfection.

\section{Luciferase reporter assay}

First, psiCHECK2-SNHG16-wt or the mutant type with miR-1285-3p mimics were co-transfected into MNNG and cells using Lipofectamine 3000 (Invitrogen; Thermo Fisher Scientific, Inc.). After $48 \mathrm{~h}$, Renilla luciferase activity was assessed and used as a measure of normalization to compare the relative luciferase activity.

\section{Xenograft transplantation}

Ten male nude $(\mathrm{BALB} / \mathrm{c})$ mice (4 weeks old, average 15 g), (which bought from Xipuer-Bikai Laboratory Animal Technology Company) were randomly divided into two groups method, and there are five mice in each group. 


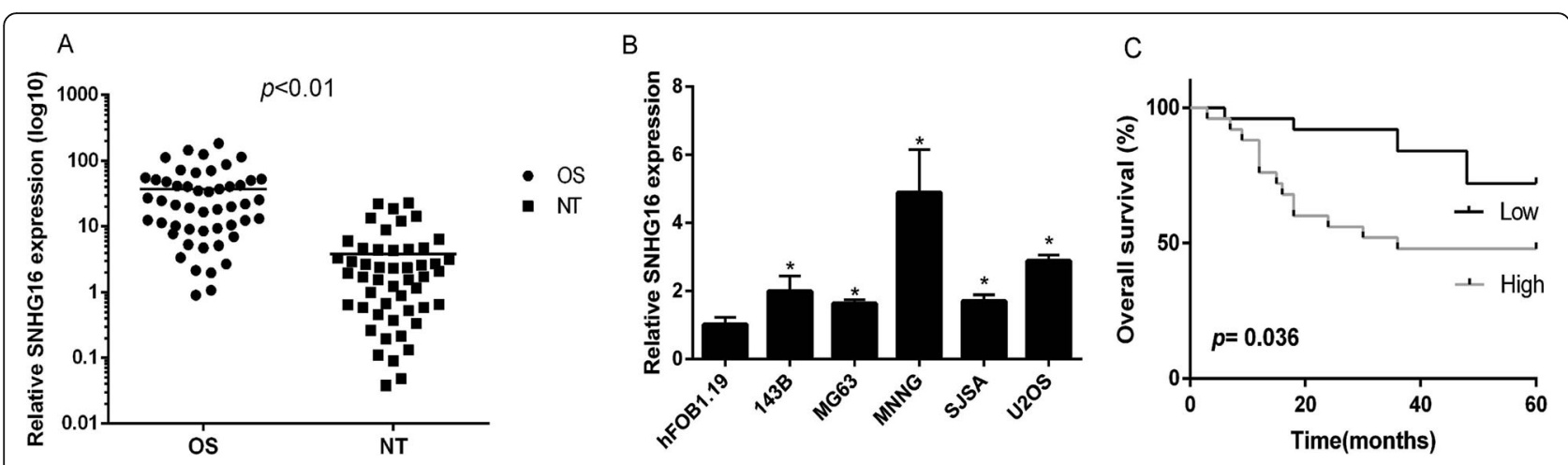

Fig. 1 SNHG16 expression in OS tissues and cell lines. (A). The SNHG16 expression in 50 pairs of OS and noncancerous tissues(NT). (B). The SNHG16 expression in five OS cell lines (U2OS, MNNG, SJSA, MG-63 and 143b) and normal osteoblast cells (hFOB1.19). (C). Kaplan-Meier analyses of the association between SNHG16 expression and overall survival $\left(^{*} p<0.05\right)$

The condition of the experiment in temperature is $22 \pm$ $2{ }^{\circ} \mathrm{C}$, and the humidity is $45-55 \% .12 \mathrm{~h}$ of light is provided per day, and each mice can eat and drink freely. By the way, we pay highly attention to the health status and activity of the mice in the cage during the study, and the room is always well ventilated, and the environment is quiet. For SNHG16, first, we transduced MNNG/HOS cells with a lentiviral vector sh-SNHG16 or sh-NC, which constitutively expressing green florescent protein (GFP) gene. Following the transduction, MNNG/HOS luciferase cells were select by the puromycin, and maintained in culture. MNNG/HOS cells stably expressing sh-SNHG16 or negative control were amplified and $1 \times 107$ cells were subcutaneously injected into the right flank of male nude (BALB/c) mice. Tumor growth and tumor volumes was measured twice a week. After 6 weeks, all mice were sacrificed by cervical spinal dislocation and tumors were excised. The animal studies were approved by the Ethics Committee of Shanghai Tenth People's Hospital.

\section{Statistical analysis}

All statistical analyses were performed using SPSS 20.0 software (IBM). Data are expressed as the Mean \pm SD for at least three separate experiments. Differences between groups were analyzed using the Student's t test or oneway ANOVA. The Kaplan-Meier method and a Cox proportional hazard regression model were used for the prognostic analysis, $p$ values $<0.05$ were considered statistically significant.

\section{Results}

SNHG16 is upregulated in OS tissues and cells, and is associated with poor prognosis

The present study performed RT-qPCR in order to determine the differential expression levels of SNHG16 in OS tissues and paired adjacent non-tumor bone tissues from 50 patients. Furthermore, the expression levels of
SNHG16 were also determined in MNNG/HOS, U2OS, MG-63, SJSA, 143b and hFOB 1.19 cells. SNHG16 expression was increased in OS tissues and cell lines compared with adjacent non-tumor bone tissues and the normal OS cell line (Fig. 1a and b). Kaplan-Meier analysis demonstrated that high expression levels of SNHG16 were significantly associated with poor prognosis (Fig. 1c). The association between clinical significance and SNHG16 expression levels in patients with OS was also analyzed. There were no significant differences between SNHG16

Table 1 Correlation between SNHG16 expression and clinicopathological features in OS patients

\begin{tabular}{|c|c|c|c|}
\hline \multirow[t]{2}{*}{ Variables } & \multicolumn{2}{|c|}{ SNHG16 expression levels } & \multirow[t]{2}{*}{$P$} \\
\hline & Low & High & \\
\hline \multicolumn{4}{|l|}{ Sex } \\
\hline Male & 17 & 12 & 0.152 \\
\hline Female & 8 & 13 & \\
\hline \multicolumn{4}{|l|}{ Age } \\
\hline$\leq 20$ & 10 & 13 & 0.395 \\
\hline$>20$ & 15 & 12 & \\
\hline \multicolumn{4}{|l|}{ Location } \\
\hline Femur/Tibia & 21 & 19 & 0.480 \\
\hline Elsewhere & 4 & 6 & \\
\hline \multicolumn{4}{|l|}{ Tumor size (cm) } \\
\hline$\leq 5$ & 8 & 12 & 0.248 \\
\hline$>5$ & 17 & 13 & \\
\hline \multicolumn{4}{|l|}{ Clinical stage } \\
\hline$I+\| A$ & 12 & 5 & 0.037 \\
\hline$\|\mathrm{B} /\| \|$ & 13 & 20 & \\
\hline \multicolumn{4}{|c|}{ Distant Metastasis } \\
\hline Yes & 7 & 9 & 0.544 \\
\hline No & 18 & 16 & \\
\hline
\end{tabular}

$P$ value was acquired by Pearson chi-square test. The median expression level was used as the cutoff 
expression and age, sex, tumor size or tumor location (Table 1). However, it was revealed that SNHG16 expression in OS was associated with clinical stage $(P=0.037)$.

\section{Knockdown of SNHG16 inhibits proliferation, migration and invasion in OS cells}

In order to investigate the functional role of SNHG16 in the progression of OS, SNHG16 siRNA and negative control were transfected into MNNG/HOS and U2OS cells. The expression level of SNHG16 was tested using RT-qPCR $48 \mathrm{~h}$ post-transfection, and the results were compared with non-specific siRNA transfection. SNHG16 siRNA transfection resulted in significant knockdown of SNHG16 expression (Fig. 2a and d). The effect of SNHG16 on OS proliferation was determined using a CCK- 8 assay. In MNNG/HOS and U2OS cells,
SNHG16 knockdown led to decreased cell proliferation compared with that in non-transfected control (NC) cells (Fig. 2b, c, e and f). Transwell assays were then used to assess the effect of SNHG16 on OS migration and invasion. As presented in Fig. 3a and b, the results revealed the positive effect of SNHG16 knockdown on MNNG/HOS and U2OS cell migration and invasion.

\section{SNHG16 blocks the cell cycle and accelerates apoptosis in OS cells}

Flow cytometry analyses were used to measure the effect of SNHG16 and miR-1285-3p on the cell cycle and apoptosis. SNHG16 knockdown in the MNNG/HOS and U2OS cells significantly increased the number of cells in the $G_{2} / M$ phase compared with the negative controls (Fig. 4C and D). Furthermore, compared with the si-NC group, knockdown

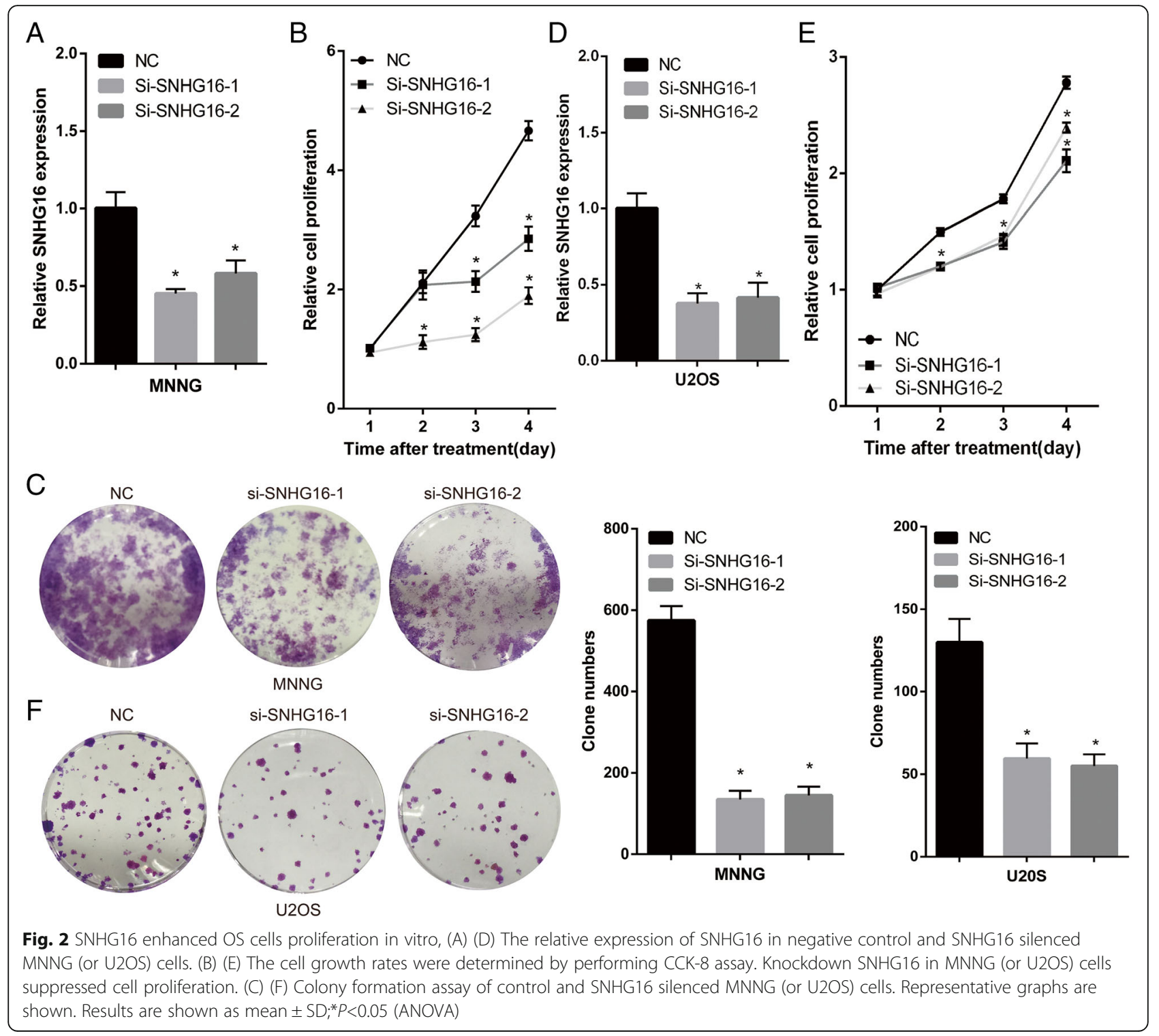


of SNHG16 resulted in an increase in the proportion of apoptotic cells in both U2OS and MNNG/HOS cells, compared with the negative controls (Fig. 4A and B).

\section{SNHG16 knockdown inhibits OS growth in vivo}

In order to demonstrate the role of SNHG16 on tumor growth in vivo, MNNG cells were stably transduced with lentivirus SNHG16-shRNA (sequence same as siSNHG16-2) and lentivirus shNC. The high GFP expression in transduced cells showed in Fig. 5A, and the transduction efficiency is presented in Fig. 5B. The mean weights and volumes of xenograft tumors generated from sh-SNHG16 MNNG cells were lower than those of shNC cells (Fig. 5C-E).

\section{SNHG16 may be a target of miR-1285-3p}

Various published articles have demonstrated that lncRNAs may act as ceRNAs through microRNA response element s(MRE) binding to miRNA. In the present study, it was investigated whether SNHG16 affected OS progression through ceRNAs and the miR-1285-3p pathway. First, bioinformatics analysis (RegRNA 2.0) was performed in order to investigate the potential combing site of SNHG16 (Fig. 6a and b), and miR-1285-3p was selected as a candidate (Fig. $6 \mathrm{c}$ and d). Secondly, the RT-qPCR analysis verified that miR-1285-3p levels were lower in OS cell lines, and could participate in OS regulation (Fig. 7). Thirdly, a luciferase reporter assay was used to assess whether miR-1285-3p could target SNHG16. According to the binding sites in SNHG16, plasmids were constructed that contained wild-type and mutant miR-1285-3p (psiCHECK2-SNHG16-wt and psiCHECK2-SNHG16-mut, respectively). The results revealed that miR-1285-3p mimics could significantly weaken the fluorescence signal of psiCHECK2-SNHG16-wt in 293cells (Fig. 6e), but no effect was observed if the binding sites in SNHG16 were mutated (co-transfection of miR1285-3p mimics and psiCHECK2-SNHG16-mut). In MNNG/HOS cells the phenomenon was the same (Fig. 6f). The expression level of SNHG16 and miR-1285-3p were then regulated in order to analyze their influence on each other. When silencing SNHG16, the expression level of miR-1285-3p was increased (Fig. 6d). Meanwhile, in the group of miR-1285-3p mimics, the rate of cells remaining in the $G_{2} / M$ phase was elevated and the apoptosis proportion was increased when compared with the group of miR1285-3p inhibitors. That is to say, the phenomenon of cell

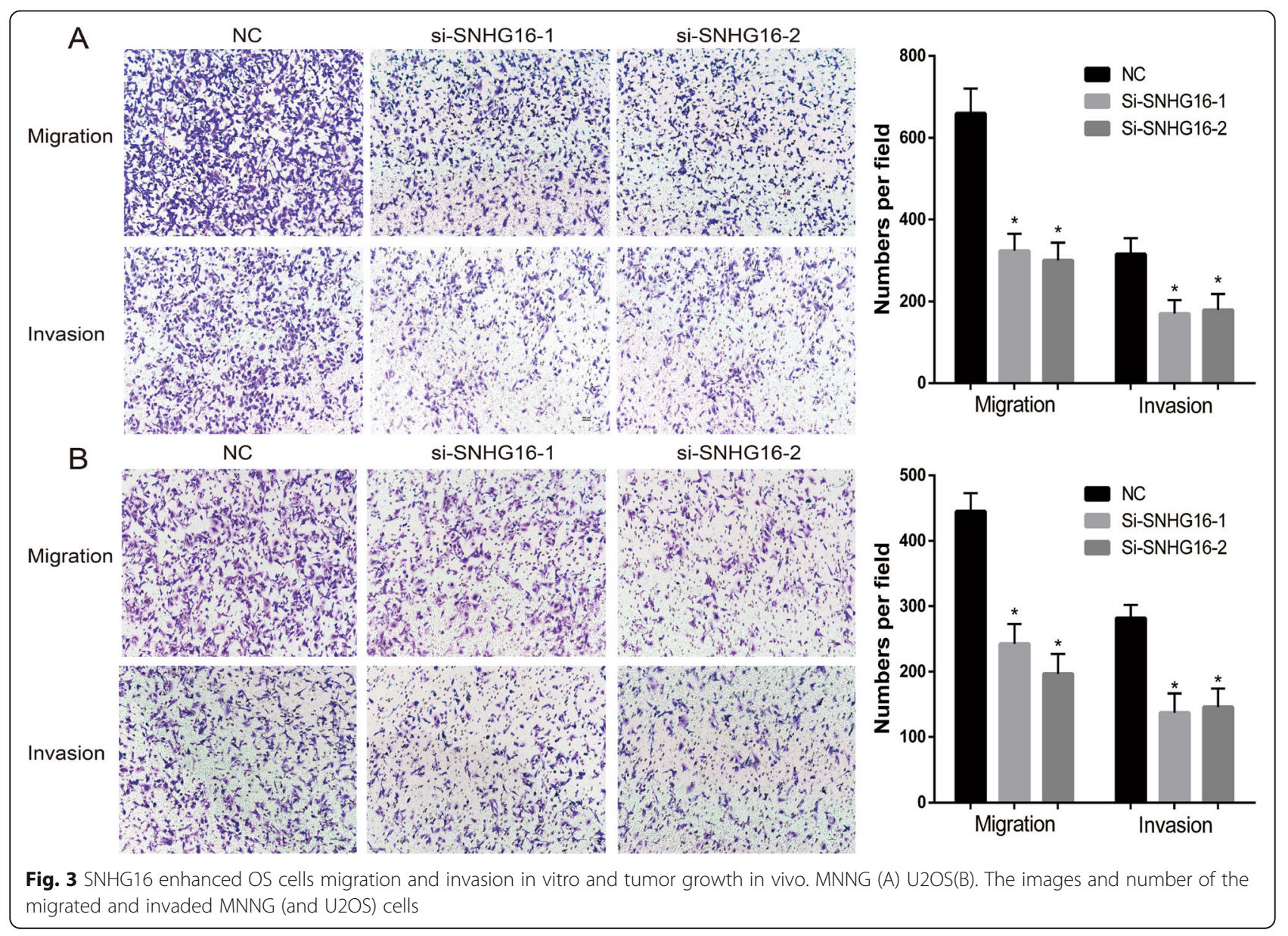




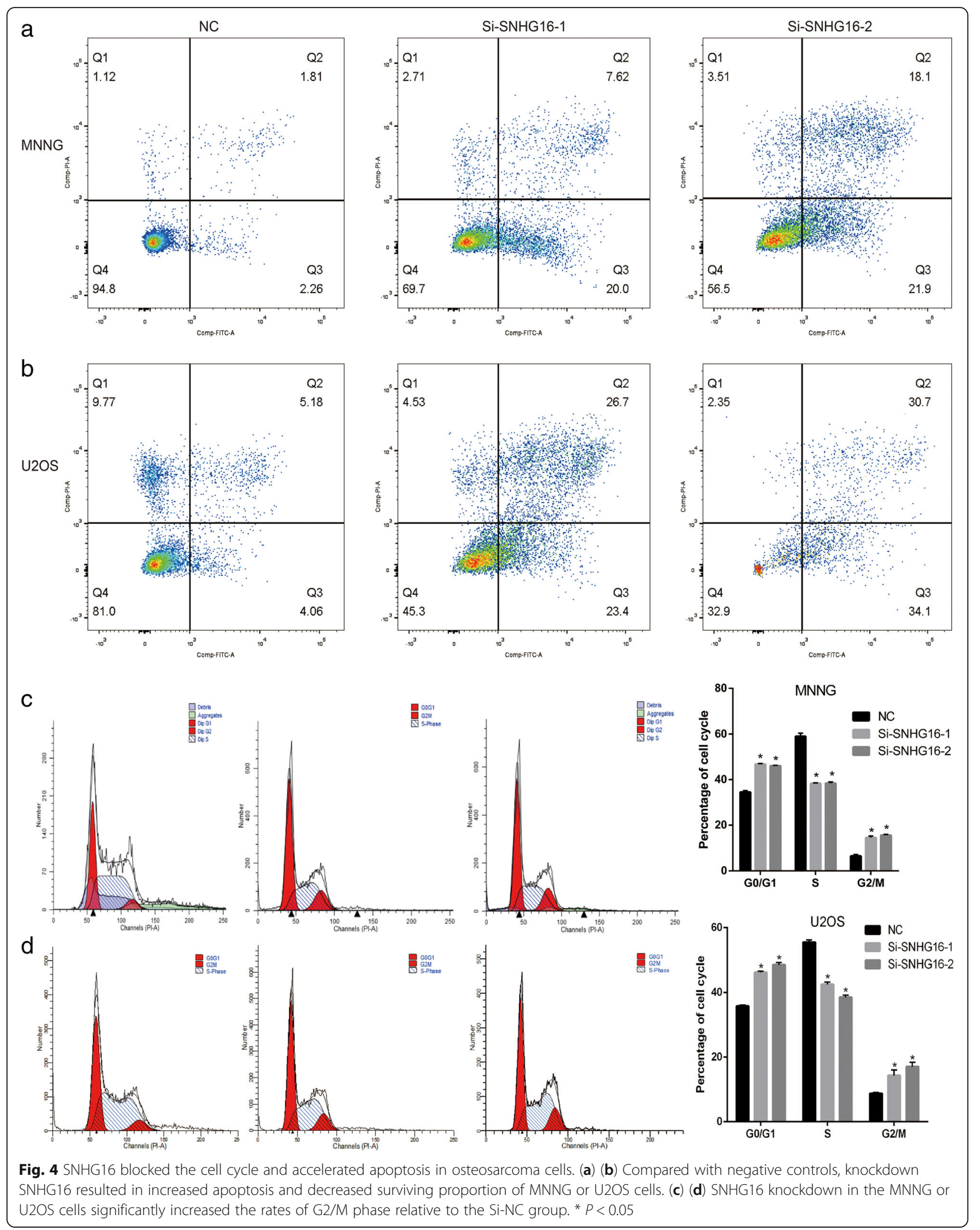




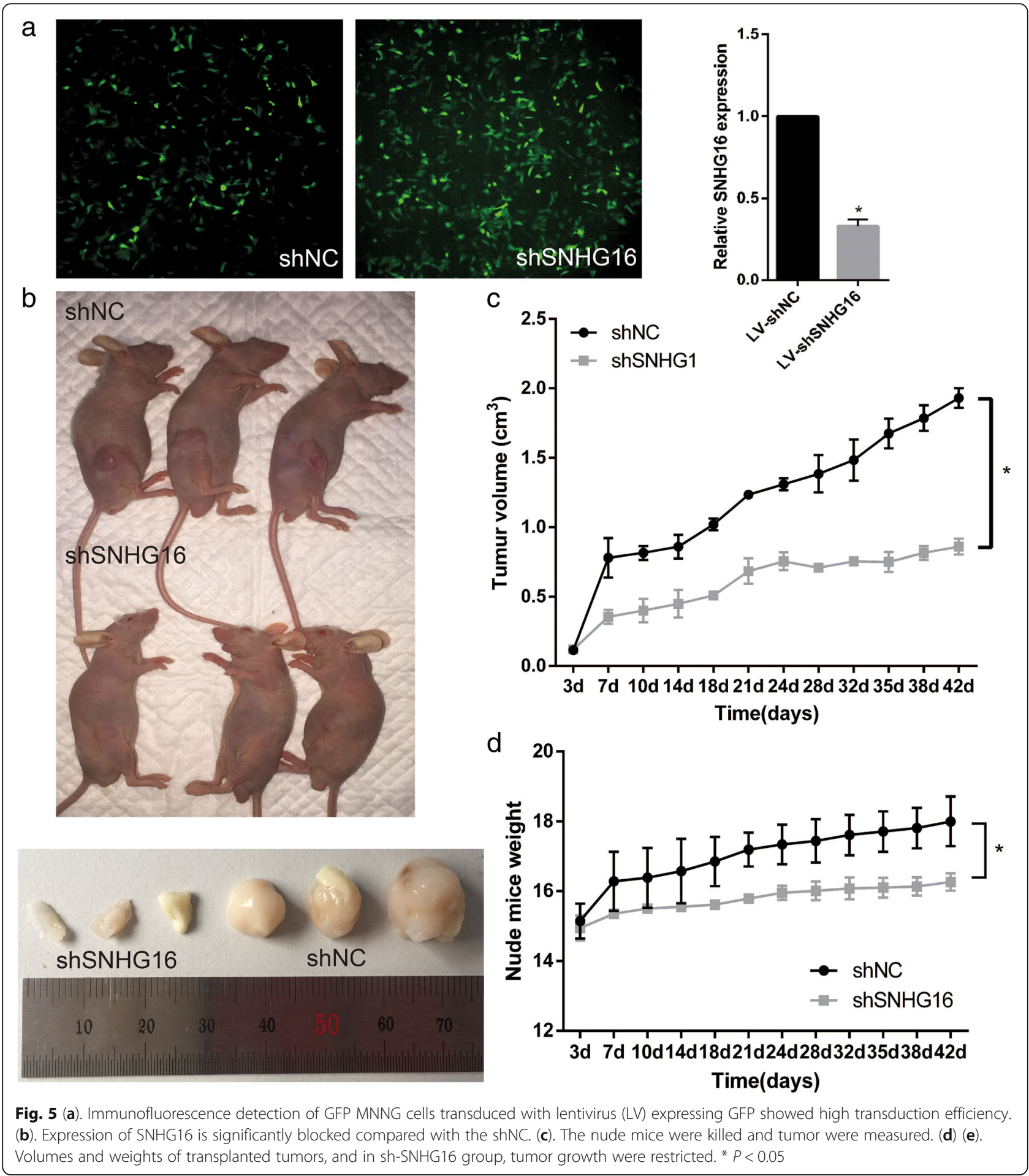

cycle and apoptosis was also negatively altered between SNHG16 and miR-1285-3p.

SNHG16 exerts its function in OS cells in a similar manner to $\mathrm{miR}-1285-3 p$

In order to determine the functional role of the interaction between SNHG16 and miR-1285-3p in OS, the present study investigated the expression level of miR1285-3p in OS cell lines (Fig. 7a), and tested the influence of miR-1285-3p in MNNG (Fig. 7b-g). Rescue experiments were then applied in order to identify the influence of SNHG16 in OS proliferation and migration through targeting miR-1285-3p. miR-NC or miR-1285$3 p$ inhibitor was transfected into shNC or shSNHG16 
cells, and the effect of SNHG16 in OS growth and migration could be partly reversed by miR-1285-3p inhibitor (Fig. 8a and b). These data illustrated that SNHG16 enhanced cell proliferation and migration via targeting miR-1285-3p.

SNHG16 silencing increases the protein expression levels of cleaved-caspase-3 and Bax, and decreases $\mathrm{Bcl}-2$ and pro-caspase-3 protein expression

Western blotting analysis was applied to measure the protein levels of cleaved-caspase-3, pro-caspase3 , Bax and Bcl-2 among the following groups. As demonstrated by the results of this analysis (Fig. 9), compared with the negative control, the sh-SNHG16 and miR-1285-3p mimics groups exhibited increased protein levels of Bax and cleaved-caspase-3, and decreased expression of pro-caspase-3 and Bcl-2. These findings revealed that silencing of SNHG16 increases the protein levels of cleaved-caspase-3 and
Bax, and decreases pro-caspase- 3 and Bcl-2 protein expression, supporting the notion that SNHG16 inhibits apoptosis by sponging miR-1285-3p in OS cells.

\section{Discussion}

Increasing evidence has confirmed that various lncRNAs are dysregulated in a number of different types of cancer, including gastric [21], lung [22], breast [23], and colorectal cancer [24]. Recent research has demonstrated that IncRNAs participate in the development of OS by regulating cell proliferation, migration, metastasis and apoptosis. For example, Perry et al [25] highlighted that the PI3K/mTOR pathway plays a fundamental part in maintaining cell viability in OS. Wang et al [26] revealed that lncRNA AK093407 promotes STAT3-mediated proliferation and inhibits OS apoptosis Zhang et al [27] demonstrated that IncRNA ODRUL regulates the progression of OS via miR-3182/MMP2 Axis Wang et al

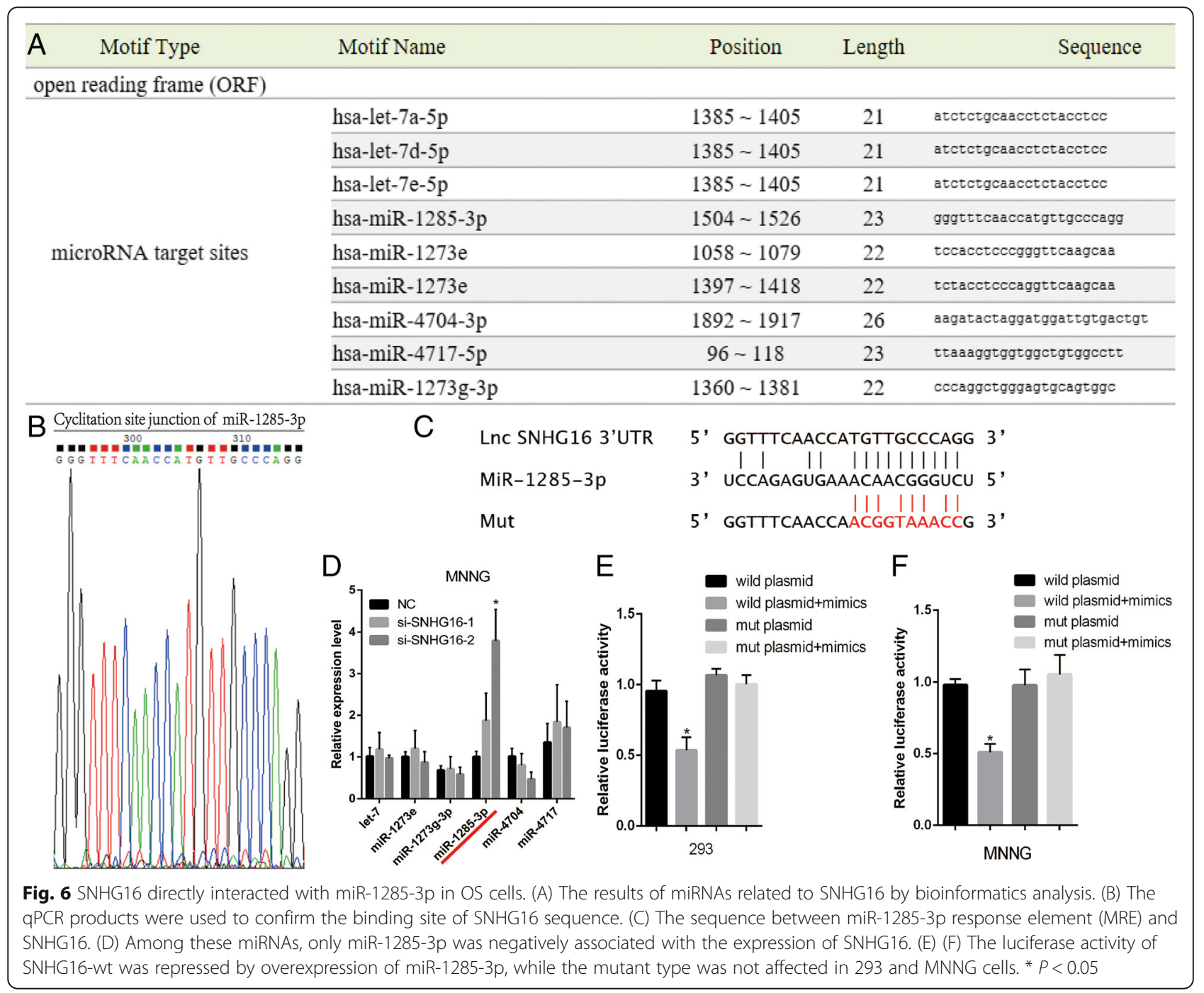




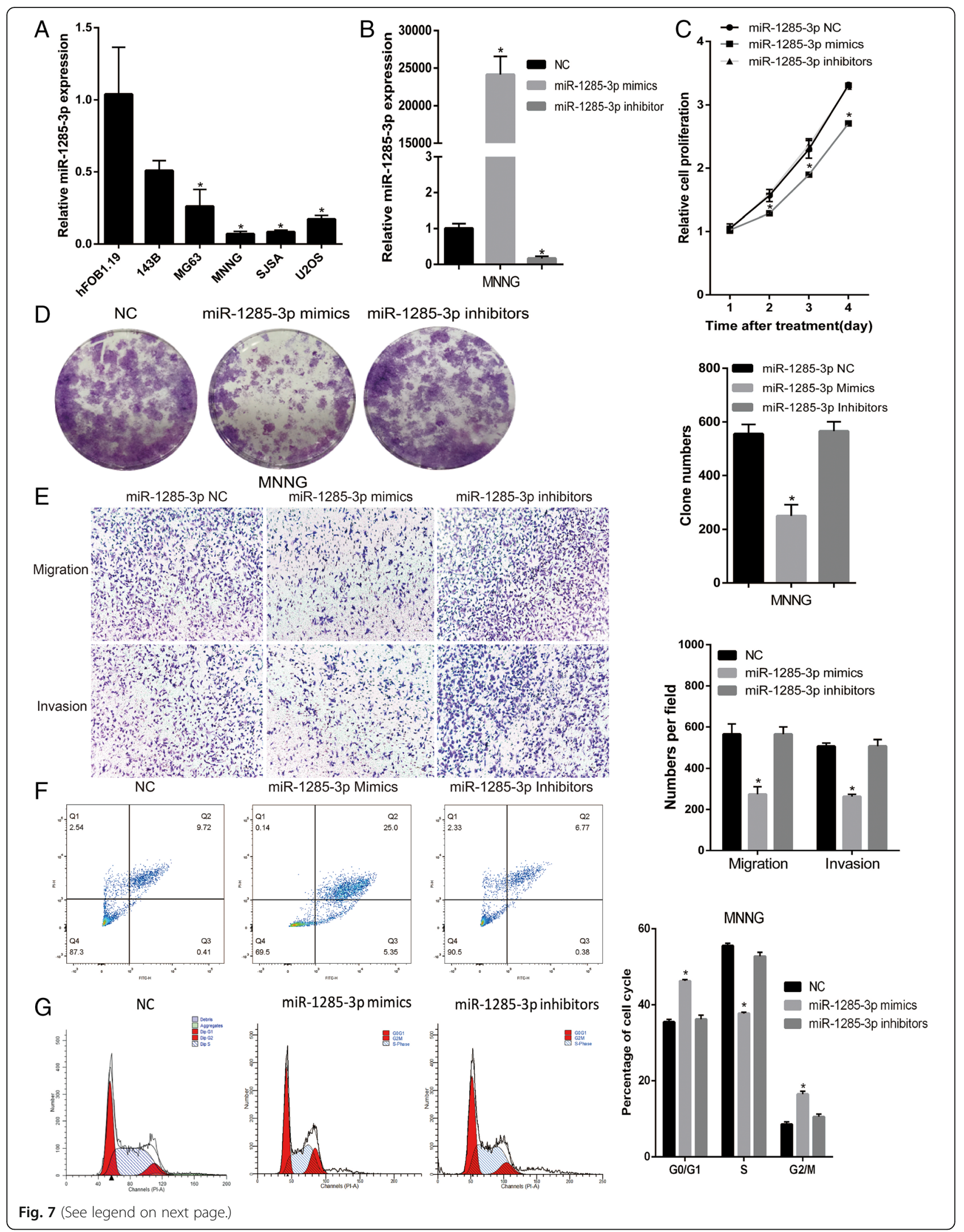


(See figure on previous page.)

Fig. 7 SNHG16 was directly targeted by miR-1285-3p. (A) The miR-1285-3p expression in five OS cell lines and a normal osteoblast cells. (B)The relative expression of miR-1285-3p mimics, inhibitors and negative control were detected in MNNG cells. (C) (D) Cell proliferation is suppressed by miR-1285-3p mimics. (E) Upregulated miR-1285-3p reduced migration and invasion of MNNG cells. (F) (G) Increasing miR-1285-3p in the MNNG cells promoted the rates of G2/M phase relative to the NC group, and enhancing expression of miR-1285-3p resulted in increased apoptosis

[28] reported that IncRNA DANCR promotes OS proliferation and metastasis via ROCK1 activation, through regulating the expression of miR-335-5p and miR-1972. SNHG16 has been reported to play a functional role in the progression of numerous different types of cancer, such as gastric cancer [29] and cervical cancer [30].

In the present study, the expression levels of a novel lncRNA SNHG16 were analyzed in OS tissues and their paired adjacent non-cancerous tissues. It was revealed that SNHG16 was increased in both OS tissues and cell lines, and was closely associated with clinical stage and poor outcome in OS. The present study indicated the functional role of SNHG16 in OS cells, in that SNHG16 knockdown could suppress cell proliferation, migration, invasion and promoted apoptosis in OS cells in vitro, as well as inhibit tumor growth in vivo; however, the regulation of SNHG16 expression remains unclear. As aforementioned, the present study first demonstrated the functional significance of SNHG16 expression in OS, and the results suggested that SNHG16 functions as an oncogene and promotes OS malignant progression. Therefore, SNHG16 may be a promising prognostic and diagnostic marker and therapeutic target for OS.

It has been suggested that lncRNAs could indirectly harbor miRNAs to decrease expression and activity by acting as a 'sponge'. Therefore, the present study performed a bioinformatics analysis and demonstrated the binding sites between SNHG16 and miR-1285-3p via a luciferase assay. The results suggested that SNHG16 may exert functions by sponging miR-1285-3p. Nevertheless, the function of miR-1285-3p in OS remains uncertain. Previous studies have demonstrated that miR-1285-3p participates in the tumorigenesis of pancreatic cancer [31] and ovarian cancer [32]. The results of the present study indicated that miR-1285-3p was significantly decreased in OS cells. Furthermore, it was demonstrated that miR-1285-3p could promote apoptosis and inhibit OS cell proliferation, migration and invasion in vitro. The present study implies that miR1285-3p may play a functional role in different types of cancer.

Overall, the present study first demonstrated that, in OS tissues and cell lines, the expression level of lncRNA SNHG16 is upregulated. High expression levels of SNHG16 in OS are closely associated with poor clinical outcome and advanced disease stage. Furthermore, it was also indicated that SNHG16 promotes OS tumor growth in vivo, OS cell invasion, migration and proliferation, and suppresses apoptosis in vitro via association with miR-1285-3p. These results validated that the interaction between SNHG16 and miR-1285-3p could be a novel pathway involved in various different types of cancer, and that this mechanism could provide a promising therapeutic target, particularly in the treatment of OS. By the way, miRNA generally acts on mRNA to regulate the transcription of target genes, and it is a limitation that the we did not study the target gene protein molecules regulated by miR-1285-3p, and we plan to test it in further investigations.

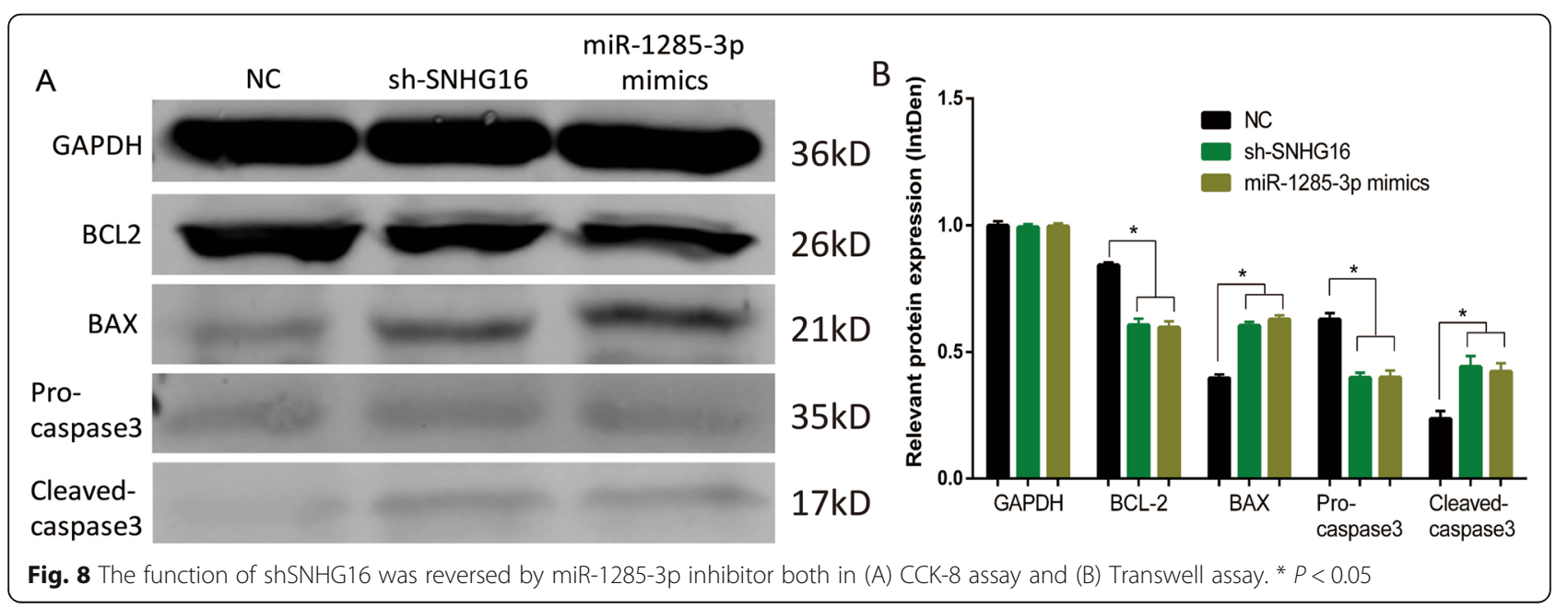




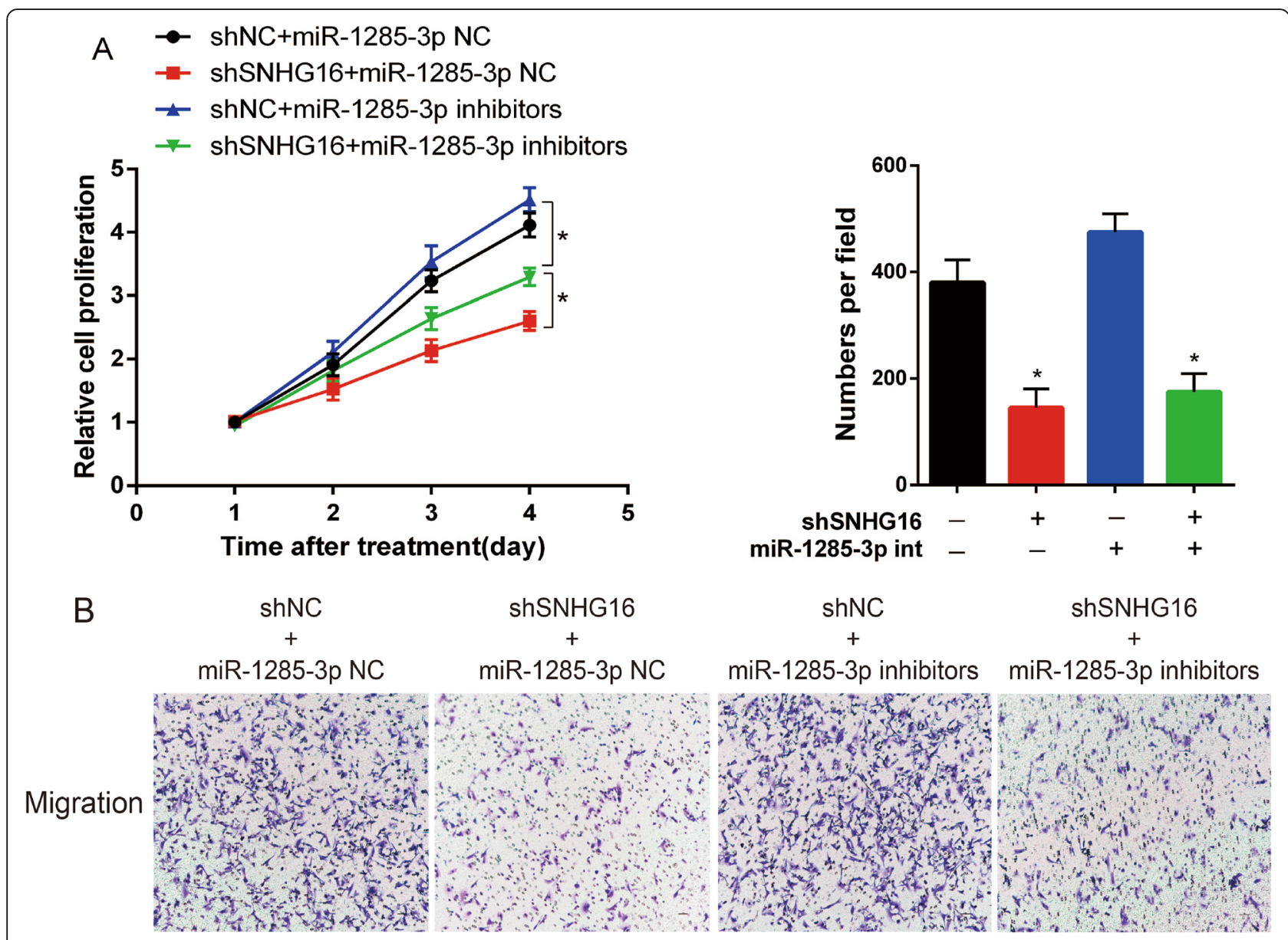

Fig. 9 (A) The protein level was shown above. (B) The density of each group. ${ }^{*} P<0.05$. Full-length blots/gels are presented in Supplementary Figure [wb origin]

\section{Conclusions}

In summary, our results from the present study demonstrate that the expression level of lncRNA SNHG16 is increased in OS cell lines compared to that in normal osteoblast cell lines. Function experiment further showed that lncRNA SNHG16 may promote the proliferation, migration, invasion and reduce apoptosis by inhibiting the expression of miR-1285-3p, which may become a novel target in OS therapy.

\section{Supplementary Information}

The online version contains supplementary material available at https://doi. org/10.1186/s12885-021-07933-2

Additional file 1. WBR

Additional file 2. Multivariate analysis

Additional file 3. GAPDH

Additional file 4. $\mathrm{BCL} 2$

Additional file 5. BAX

Additional file 6. Pro-caspase

Additional file 7. Cleaved-caspase

\section{Abbreviations}

LncRNAs: Long non-coding RNAs; SNHG16: Small nucleolar RNA host gene 16; OS: osteosarcoma; CCK-8: Cell Counting Kit-8; ceRNA: Competing endogenous RNA; RT-Qpcr: Reverse transcription-quantitative Polymerase Chain Reaction; OD: Optical density; GFP: Green florescent protein; GAPD H: Glyceraldehyde-3-phosphate dehydrogenase; Bcl2: B-cell lymphoma-2; BAX: BCI2-Associated X

Acknowledgements

Not applicable.

Authors' contributions

XX and GJ conceived and designed the study. SH and YSF performed the experiments. STZ provided the samples. GL and HYY analyzed the data. SSH reviewed and edited the manuscript. All authors have read and approved the manuscript.

Funding

No funding was received.

Availability of data and materials

The datasets used or analyzed during the present study are available from the first author or corresponding author upon reasonable request.

Ethics approval and consent to participate

The present study was approved by the Ethics Committee of Shanghai Tenth People's Hospital. 


\section{Consent for publication}

Not applicable.

\section{Competing interests}

The authors declare that they have no competing interests.

\section{Author details}

'Department of Orthopedic, Shanghai Tenth People's Hospital, Tongji University School of Medicine, Shanghai 200072, China. ${ }^{2}$ Spinal Pain Research Institute, Tongji University School of Medicine, Shanghai 200072, China. ${ }^{3}$ Department of Hematology, Shanghai Institute of Hematology, Ruijin Hospital affiliated to School of Medicine, Shanghai Jiaotong University School of Medicine, Shanghai 200025, China. ${ }^{4}$ Department of Orthopedic, The First Affiliated Hospital of Nanchang University, Nanchang 330006, Jiangxi Province, China.

Received: 21 August 2020 Accepted: 18 February 2021 Published online: 06 April 2021

\section{References}

1. Isakoff MS, Bielack SS, Meltzer P, Gorlick R. Osteosarcoma: current treatment and a collaborative pathway to success. J Clin Oncol. 2015;33(27):3029-35.

2. Gianferante DM, Mirabello L, Savage SA. Germline and somatic genetics of osteosarcoma - connecting aetiology, biology and therapy. Nat Rev Endocrinol. 2017:13(8):480-91.

3. Zhang $\mathrm{CL}$, Zhu KP, Ma XL. Antisense IncRNA FOXC2-AS1 promotes doxorubicin resistance in osteosarcoma by increasing the expression of FOXC2. Cancer Lett. 2017;396:66-75.

4. Flynn RA, Chang HY. Long noncoding RNAs in cell-fate programming and reprogramming. Cell Stem Cell. 2014;14(6):752-61.

5. Shi $X$, Sun M, Liu H, Yao Y, Song Y. Long non-coding RNAs: a new frontier in the study of human diseases. Cancer Lett. 2013;339(2):159-66.

6. Schmitt AM, Chang HY. Long noncoding RNAs in Cancer pathways. Cancer Cell. 2016;29(4):452-63.

7. Ju C, Zhou R, Sun J, Zhang F, Tang X, Chen KK, Zhao J, Lan X, Lin S, Zhang $Z$, et al. LncRNA SNHG5 promotes the progression of osteosarcoma by sponging the miR-212-3p/SGK3 axis. Cancer Cell Int. 2018;18:141.

8. Liu B, Zhao H, Zhang L, Shi X. Silencing of long-non-coding RNA ANCR suppresses the migration and invasion of osteosarcoma cells by activating the p38MAPK signalling pathway. BMC Cancer. 2019;19(1):1112.

9. Yu M, Ohira M, Li Y, Niizuma H, Oo ML, Zhu Y, Ozaki T, Isogai E, Nakamura Y, Koda T, et al. High expression of ncRAN, a novel non-coding RNA mapped to chromosome 17q25.1, is associated with poor prognosis in neuroblastoma. Int J Oncol. 2009;34(4):931-8.

10. Wang X, Liu L, Zhao W, Li Q, Wang G, Li H. LncRNA SNHG16 promotes the progression of laryngeal squamous cell carcinoma by mediating miR-8775p/FOXP4 Axis. Onco Targets Ther. 2020;13:4569-79.

11. Cao X, Xu J, Yue D. LncRNA-SNHG16 predicts poor prognosis and promotes tumor proliferation through epigenetically silencing p21 in bladder cancer. Cancer Gene Ther. 2018;25(1-2):10-7.

12. Han GH, Lu KJ, Wang P, Ye J, Ye YY, Huang JX. LncRNA SNHG16 predicts poor prognosis in ESCC and promotes cell proliferation and invasion by regulating Wnt/beta-catenin signaling pathway. Eur Rev Med Pharmacol Sci. 2018;22(12):3795-803.

13. Zhang $G$, Ma A, Jin $Y$, Pan G, Wang C. LncRNA SNHG16 induced by TFAP2A modulates glycolysis and proliferation of endometrial carcinoma through miR-490-3p/HK2 axis. Am J Transl Res. 2019;11(11):7137-45.

14. Zhu H, Zeng Y, Zhou CC, Ye W. SNHG16/miR-216-5p/ZEB1 signal pathway contributes to the tumorigenesis of cervical cancer cells. Arch Biochem Biophys. 2018;637:1-8.

15. Christensen LL, True K, Hamilton MP, Nielsen MM, Damas ND, Damgaard CK, Ongen H, Dermitzakis E, Bramsen JB, Pedersen JS, et al. SNHG16 is regulated by the Wnt pathway in colorectal cancer and affects genes involved in lipid metabolism. Mol Oncol. 2016;10(8):1266-82.

16. Zhu Y, Yu M, Li Z, Kong C, Bi J, Li J, Gao Z, Li Z. ncRAN, a newly identified long noncoding RNA, enhances human bladder tumor growth, invasion, and survival. Urology. 2011;77(2):510 e511-5.

17. Fabbri M, Croce CM, Calin GA. MicroRNAs. Cancer J. 2008;14(1):1-6.

18. Hidaka H, Seki N, Yoshino H, Yamasaki T, Yamada Y, Nohata N, Fuse M, Nakagawa M, Enokida H. Tumor suppressive microRNA-1285 regulates novel molecular targets: aberrant expression and functional significance in renal cell carcinoma. Oncotarget. 2012;3(1):44-57.

19. Hironaka-Mitsuhashi A, Otsuka K, Gailhouste L, Sanchez Calle A, Kumazaki M, Yamamoto Y, Fujiwara Y, Ochiya T. MiR-1285-5p/TMEM194A axis affects cell proliferation in breast cancer. Cancer Sci. 2020;111(2):395-405.

20. Salmena L, Poliseno L, Tay Y, Kats L, Pandolfi PP. A ceRNA hypothesis: the Rosetta stone of a hidden RNA language? Cell. 2011;146(3):353-8.

21. Wu DC, Wang SSW, Liu CJ, Wuputra K, Kato K, Lee YL, Lin YC, Tsai MH, Ku CC, Lin WH, et al. Reprogramming antagonizes the Oncogenicity of HOXA13-long noncoding RNA HOTTIP Axis in gastric Cancer cells. Stem Cells. 2017:35(10):2115-28.

22. Wu D, Yang B, Chen J, Xiong H, Li Y, Pan Z, Cao Y, Chen J, Li T, Zhou S, et al. Upregulation of long non-coding RNA RAB1A-2 induces FGF1 expression worsening lung cancer prognosis. Cancer Lett. 2018;438:116-25.

23. Gu J, Wang $Y$, Wang $X$, Zhou D, Shao C, Zhou M, He Z. Downregulation of IncRNA GAS5 confers tamoxifen resistance by activating miR-222 in breast cancer. Cancer Lett. 2018;434:1-10.

24. Qin W, Wang X, Wang Y, Li Y, Chen Q, Hu X, Wu Z, Zhao P, Li S, Zhao H, et al. Functional polymorphisms of the IncRNA H19 promoter region contribute to the cancer risk and clinical outcomes in advanced colorectal cancer. Cancer Cell Int. 2019;19:215.

25. Perry JA, Kiezun A, Tonzi P, Van Allen EM, Carter SL, Baca SC, Cowley GS, Bhatt AS, Rheinbay E, Pedamallu CS, et al. Complementary genomic approaches highlight the PI3K/mTOR pathway as a common vulnerability in osteosarcoma. Proc Natl Acad Sci U S A. 2014;111(51):E5564-73.

26. Wang Y, Liang T, Wang Y, Huang Y, Li Y. Long non-coding RNA AK093407 promotes proliferation and inhibits apoptosis of human osteosarcoma cells via STAT3 activation. Am J Cancer Res. 2017;7(4):892-902.

27. Zhu KP, Ma XL, Zhang CL. LncRNA ODRUL contributes to osteosarcoma progression through the miR-3182/MMP2 Axis. Mol Ther. 2017;25(10):238393.

28. Wang $Y$, Zeng $X$, Wang N, Zhao W, Zhang $X$, Teng S, Zhang Y, Lu Z. Long noncoding RNA DANCR, working as a competitive endogenous RNA, promotes ROCK1-mediated proliferation and metastasis via decoying of miR-335-5p and miR-1972 in osteosarcoma. Mol Cancer. 2018;17(1):89.

29. Zhou C, Zhao J, Liu J, Wei S, Xia Y, Xia W, Bi Y, Yan Z, Huang H. LncRNA SNHG16 promotes epithelial- mesenchymal transition via down-regulation of DKK3 in gastric cancer. Cancer Biomark. 2019;26(4):393-401.

30. Tao L, Wang X, Zhou Q. Long noncoding RNA SNHG16 promotes the tumorigenicity of cervical cancer cells by recruiting transcriptional factor SPI1 to upregulate PARP9. Cell Biol Int. 2020;44(3):773-84.

31. Huang $H$, Xiong $G$, Shen $P$, Cao Z, Zheng L, Zhang T, Zhao Y. MicroRNA1285 inhibits malignant biological behaviors of human pancreatic cancer cells by negative regulation of YAP1. Neoplasma. 2017;64(3):358-66.

32. Zhang Y, Ruan F. LncRNA LEF1-AS1 promotes ovarian Cancer development through interacting with miR-1285-3p. Cancer Manag Res. 2020;12:687-94.

\section{Publisher's Note}

Springer Nature remains neutral with regard to jurisdictional claims in published maps and institutional affiliations.

Ready to submit your research? Choose BMC and benefit from:

- fast, convenient online submission

- thorough peer review by experienced researchers in your field

- rapid publication on acceptance

- support for research data, including large and complex data types

- gold Open Access which fosters wider collaboration and increased citations

- maximum visibility for your research: over $100 \mathrm{M}$ website views per year

At $\mathrm{BMC}$, research is always in progress.

Learn more biomedcentral.com/submission 